\title{
Cosmological simulations of structure formation and the Vlasov equation
}

\author{
Michael Joyce \\ Laboratoire de Physique Nucléaire et de Hautes Energies, \\ Université Pierre et Marie Curie-Paris 6, UMR 7585, \\ Paris, F-75005 France.
}

\begin{abstract}
In cosmology numerical simulations of structure formation are now of central importance, as they are the sole instrument for providing detailed predictions of current cosmological models for a whole class of important constraining observations. These simulations are essentially molecular dynamics simulations of $\mathrm{N}$ ( $\gg 1$, now up to of order several billion) particles interacting through their self-gravity. While their aim is to produce the Vlasov limit, which describes the underlying ("cold dark matter") models, the degree to which they actually do produce this limit is currently understood, at best, only very qualitatively, and there is an acknowledged need for "a theory of discreteness errors". In this talk I will describe, for non-cosmologists, both the simulations and the underlying theoretical models, and will then focus on the issue of discreteness, describing some recent progress in addressing this question quantitatively.
\end{abstract}

Key words: Vlasov-Poisson, cosmological N-body simulations, discreteness effects. PACS: 98.80.-k,95.10.Ce

\section{Introduction}

Cosmology is a branch of physics which has been extremely dynamic in the last decade or so. This dynamism has come essentially from the explosion of new data constraining cosmological models, from a whole range of observational techniques. Most important of all are, without doubt, the observations of the "three degree Kelvin" cosmic microwave background radiation (CMBR): there now exist detailed maps of the tiny fluctuations in the temperature of this radiation as a function of direction on the sky, at an angular resolution well below a degree with a sensitivity of less than $100 \mu \mathrm{K}$ [WMAP collaboration

Email address: joyce@lpnphep.in2p3.fr (Michael Joyce). 
(2006)]. This is the "relic" radiation which decoupled (electromagnetically) from matter when the universe was a few hundred thousand years old. These maps of temperature fluctuations thus provide very precise constraints on the spatial fluctuations in the energy density in the universe at this time, which may in turn be related (through the Poisson equation, suitably modified in the context of general relativity) to the fluctuations in the gravitational potential at this time. In the paradigm defined by current cosmological models these very low amplitude fluctuations at "early" times develop by gravitational instability, giving rise ultimately to the highly inhomogeneous universe observed today: the distribution of galaxies and galaxy clusters which shows a high degree of inhomogeneity with structures extending over a significant fraction of the visible universe [see e.g. Gabrielli et al. (2005)]. This is the problem of "large scale structure formation", one of the central challenges in cosmology today.

The specific model of the universe which cosmologists have developed to account for current observations posits that the dominant fraction (more than eighty per cent) of the gravitating matter in the universe is so called "cold dark matter" (CDM). This is matter with small velocity dispersion (and therefore non-relativistic) which interacts only gravitationally (or almost only). Further on the scales of interest the approximation of Newtonian gravity is very good. Thus the problem of structure formation can be reduced, to a good approximation, to that of self-gravitating particles in an infinite space evolving from some given initial conditions (see below) ${ }^{1}$. These initial conditions (constrained as we have noted by the CMBR) are what we can call quasi-uniform, i.e., they exhibit small deviations from an exactly uniform distribution of constant density, fluctuations averaged in a sphere about the mean density typically decaying away as some power of the radius.

The CDM particles are, further, usually posited to be microscopic, with masses of the order of those of particles in the standard model of particles physics (i.e. within a few orders of magnitude of the proton mass). The macroscopic scales on which fluctuations are probed by cosmological observations thus contain a huge number of such particles (e.g. of order $10^{70}$ to $10^{80}$ in standard models). This suggests that the relevant dynamics of the CDM can be well approximated by the Vlasov limit, taking the particle number $N$ to infinity in an appropriate manner. In fact it is assumed canonically in cosmology that the correct dynamical description of CDM is given by the coupled Vlasov-Poisson equations:

$$
\begin{aligned}
& \frac{\partial f(\mathbf{r}, \mathbf{v}, t)}{\partial t}+\mathbf{v} \cdot \nabla f(\mathbf{r}, \mathbf{v}, t)-\nabla \Phi \cdot \frac{\partial f(\mathbf{r}, \mathbf{v}, t)}{\partial \mathbf{v}}=0 \\
& \nabla^{2} \Phi(\mathbf{r}, t)=4 \pi G\left[\int d^{3} v f(\mathbf{r}, \mathbf{v}, t)-\rho_{0}\right]
\end{aligned}
$$

where $f(\mathbf{r}, \mathbf{v}, t)$ is the smooth one-particle phase space density (i.e. the mass per unit phase space volume), $G$ the gravitational constant, $\Phi$ the gravitational potential and $\rho_{0}$ the mean mass density.

In the literature on cosmology and astrophysics the use of the Vlasov-Poisson system is usually very qualitatively justified using simple considerations about time scales for

\footnotetext{
1 The expansion of the background space-time, which is taken into account in cosmological simulations, leads only to a very simple modification (see below) of the Newtonian equations of motion in a static space-time.
} 
two body collisions [see e.g. Binney \& Tremaine (1987)]. Authors who give derivations mostly proceed [e.g. Peebles (1980); Saslaw (2000)] through a truncation of the BBGKY hierarchy for the full $\mathrm{N}$ particle phase space density, but one by Buchert \& Dominguez (2004) is closely inspired by the approach [see, e.g., Spohn (1991)] probably more familiar to the audience of this conference, which uses a coarse-graining of the exact single particle "spiky" phase space density which obeys a Liouville equation. These derivations of the Vlasov-Poisson limit for the purely self-gravitating CDM system in this context are not rigorous: they show that the equations (1) may be obtained in a systematic manner when an infinite number of other terms are negligible. They do not show rigorously, however, that the corresponding regime actually exists, nor that it is the one describing the CDM limit. We will not address this question here which may, however, be of interest to participants at this conference ${ }^{2}$.

A central theoretical problem in cosmology is thus the solution of this set of VlasovPoisson equations starting from given initial conditions. Analytically they have so far proved intractable albeit for some very trivial cases. Using various perturbative approaches [see e.g. Peebles (1980)], however, simple general results can be derived. The most important such result is that, at leading order in a linearisation of the density and velocity perturbation fields, the amplitude of the fluctuations in the density field, analysed in Fourier space, grow in a simple way, independent of the wavelength, as a function of time ${ }^{3}$. This approach can be extended to higher order, but breaks down inevitably when the density fluctuations become large.

\section{Dissipationless $\mathbf{N}$ body simulations}

Beyond the perturbative regime just described numerical simulations are essentially the sole instrument used to model the evolution of CDM, and hence for extracting detailed predictions of the distribution of matter in the universe today for confrontation with data ${ }^{4}$. These simulations, however, do not attempt to solve the Vlasov-Poisson directly : it is simply not numerically feasible to do so because of the phase space resolution necessary. Structures form under gravity by the collapse of overdensities. To be useful in cosmology simulations must resolve, at the very least, two decades in space and momentum, which translates (in three dimensions) to treating the long-range interaction on a grid with $10^{12}$ points. Even if clever algorithms allow one to reduce the number of operations at each step to much less than the square of the number of grid points, this is too much to handle even with current supercomputers ${ }^{5}$.

Instead of tackling the problem directly, cosmologists solve numerically the much simpler problem of the evolution of $\mathrm{N}$ self-gravitating bodies, where $N$ is as large a number as feasible. In the first generation of CDM simulations in the 1980s $N$ was of the order of a few tens of thousands, but it has increased continuously to attain almost ten billion in recent simulations! We now describe very briefly these simulations and outline, qual-

\footnotetext{
2 See contribution of M. Kiessling.

3 See the contribution of B. Marcos (arXiv:0805.1500) for more detail.

4 We discuss in this paper solely the full three dimensionsal case as in cosmology. Studies of the analagous one-dimensional system, which show qualitatively similar features [see, e.g., Rouet et al. (1990); Miller \& Rouet (2002)], may be useful for clarifying some of the issues discussed here.

5 See the contribution of S. Colombi.
} 
itatively, some of the results which they have given. We then turn to the "problem of discreteness", which is simply that of the relation between the results of these simulations and the solutions of the Vlasov-Poisson system they attempt to model.

\subsection{Methods}

We consider what are known as "dissipationless CDM simulations", which are an important class of simulation used to make predictions of the current CDM cosmological models. They are so called neglect the dissipative processes which come into play primarily because of the gravitational coupling of CDM to ordinary matter. The essential features of these N-body simulations (NBS) are the following [for reviews see e.g. Bagla \& Padmanabhan (1997); Bertschinger (1998)] :

- One considers $N$ particles in a cubic box of side $L$ with periodic boundary conditions.

- The equations of motion which are solved numerically are:

$$
\ddot{\mathbf{x}}_{i}+2 \frac{\dot{a}}{a} \dot{\mathbf{x}}_{i}=-\frac{1}{a^{3}} \sum_{i \neq j} \nabla_{x_{i}} V\left(\left|\mathbf{x}_{i}-\mathbf{x}_{j}\right|\right),
$$

where $\mathbf{x}_{i}$ is the "comoving" position of the $i$-th particle, and $a(t)$ is the "scale factor" for the cosmological model considered. The latter is a monotonically increasing function of the time, and grows by a factor of $50-100$ in a typical simulation. The two-body potential $V\left(\left|\mathbf{x}_{i}-\mathbf{x}_{j}\right|\right)$ of the particles is the Newtonian potential, with a regularisation of the singular ultra-violet behaviour at small scales. By "small" scales we mean that the characteristic scale of the smoothing $\varepsilon$ is much smaller than the initial mean interparticle distance ${ }^{6}$.

- The initial positions and velocities of the particles are determined from the initial conditions (IC) of the theoretical model using the so-called "Zeldovich approximation". This is an approximation to the evolution of a self-gravitating fluid which gives the displacements of fluid elements from a uniform state, and their velocities, in terms of the local gravitational field. The latter may be determined, and thus the displacement and velocity fields, for any set of initial density fluctuations (using the Poisson equation). The theoretical density fluctuations are canonically Gaussian, and thus fully specified by their power spectrum ${ }^{7}$ (PS). A realization of this PS is thus used to generate the displacements and velocities, and these are applied to the particles of the $\mathrm{N}$ body simulation, placed in a "uniform" configuration usually taken to be a simple cubic lattice (or sometimes "glassy" configuration).

\subsection{Results}

Different approaches to characterising the results of $\mathrm{N}$ body simulations may be found in the cosmological literature. While the early literature concentrated almost exclusively on two point correlation properties measured in real space, subsequently reciprocal space

\footnotetext{
${ }^{6}$ The exact form of the smoothing used depends on the code, and the canonical choice has evolved. Current codes typically have the exact Newtonian potential above $\varepsilon$.

7 This is equivalent, up to a normalization, to what is called the "structure factor" in solid state physics.
} 
properties (notably the PS which is simply the Fourier transform of the two point correlation function) became more widely used. These extensive (and continuing) studies have led essentially to various phenomenological ansatzes which express the final two point correlation properties as a functional of the initial two point correlation properties [Peacock \& Dodds (1996)]. More recently an alternative description of results in terms of a different phenomenological description, the so-called "halo-model" has become popular [for a review see Cooray \& Sheth (2002)]. This is based on the fact that the structures formed in simulations are observed to be well described as a collection of virialised clumps, whose masses and spatial correlations may be determined from the initial conditions, but whose internal organisation seems to have universal properties (i.e. independent of the initial conditions).

\section{The problem of discreteness}

As in any numerical simulation errors arise from the discretisation of the equations of motion intrinsic to the method. This is not what we mean by the problem of discreteness in cosmological NBS, which arises from the intrinsic difference between the simulated N-body system and the physical system of a much larger number of particles, which is supposed to be described, as we have discussed above, by the Vlasov-Poisson equations. Concretely the question can be put as follows: what is the difference, at any given time and spatial scale, between, say, a two-point correlation function measured in a perfect $N$ body simulation and the same quantity in the modelled Vlasov-Poisson system? This question is, perhaps surprisingly, one which has been relatively neglected by cosmologists, and several of the few controlled studies which exist [see e.g. Splinter et al. (1998)] raise questions about the quite optimistic assumptions widely made by simulators in linking their results of theory. It has become an issue of increasing importance in the last few years - and will take on more importance inevitably in the coming years — as much greater precision predictions from simulations is now required to exploit observational advances.

In work over the last two years we have tried to approach this question in a systematic manner. Our work can be divided into three parts, the first two of which are essentially completed while the third remains under investigation.

\subsection{Discreteness in initial conditions}

It is evidently important to understand the relation between the continuous theoretical model and the discrete simulated system already at the starting time. We have thus considered the question of the relation between the statistical properties of the density fluctuations in the initial conditions(IC) and those of the theoretical input model. As explained above, the IC are generated by displacing the particles off a perfect lattice in a manner prescribed by an input PS. Using a general formalism developed in Gabrielli (2004), we have determined precisely [Joyce \& Marcos (2004)] the PS of the IC of cosmological simulations. To a first approximation this is essentially just the sum of the PS of the underlying lattice, the input PS and a convolution term. For wavenumbers below that corresponding to the Nyquist frequency of the lattice, the full PS is well approximated by the input one. In direct (real) space the situation is very different because the Fourier 
transform of the lattice PS is a highly delocalised function, which always dominates over that of the input PS for sufficiently low amplitude of the latter. This means that, in the limit of low amplitude, the discretised system becomes a poor representation in real space of the continuous model, while remaining good in most of reciprocal space. Cosmologists usually analyse the initial conditions only in reciprocal space, and a priori it is not clear whether our finding has dynamical relevance. It turns out, as we will now explain, that it does.

\subsection{Early time evolution: A perturbative approach}

NBS begin, by construction, in the regime in which the Zeldovich approximation is valid, which means that the particles are initially close to the lattice configuration. In this case we have found ${ }^{8}$ that one can generalise usefully the standard procedure employed to describe the perturbations to a crystal in solid state physics: one linearizes the force in the displacements and determines the eigenmodes and eigenvalues of the $3 N \times 3 N$ matrix. Each such eigenmode is labelled by a reciprocal lattice vector $\mathbf{k}$, and the sign of the eigenvalues determines whether a mode grow or oscillates. We have shown [Joyce et al. (2005); Marcos et al. (2006)] how one can recover the equivalent perturbative regime of the fluid limit derived from the Vlasov-Poisson system from such a treatment, in the limit $k \ell \rightarrow 0$ (where $k$ is the modulus of $\mathbf{k}$, and $\ell$ is the lattice spacing). Having recovered this limit we can then quantify precisely the differences between the evolution of the continuous model and of its $\mathrm{N}$ body discretisation, i.e., the discreteness effects in the regime of validity of the perturbative approximation. One important result which is found is that such differences grow in time, i.e., the $\mathrm{N}$ body dynamics always diverge from the Vlasov dynamics for sufficiently long times. This is probably a result unsurpising to many of those at this conference, but it was unknown in the context of simulations of gravity in cosmology: the temporal duration of a simulation is not a parameter which has been considered in testing for discreteness effects ${ }^{9}$. We note that in the limit of low amplitude of the initial PS, this perturbative approximation for the dynamics remains valid for an arbitrarily long in time. Thus the divergence in this former limit of the discretised IC from the theoretical IC, which we described above, translates also into a divergence of the evolved discrete system from its continuum counterpart.

\subsection{Evolution in the non-linear regime}

All cosmological NBS aim to go well beyond the perturbative regime, and ultimately it is the role of discreteness in this regime which we must understand. The problem is evidently intractable analytically insofar as it poses an even more difficult one that the (analytically intractable) Vlasov-Poisson system. One can, however, approach the problem numerically in various ways. Controlled tests for effects explicitly due to discreteness in the system may be defined. For example, one can investigate the role of interactions between nearest neighbour particles which should become subdominant in the Vlasov

\footnotetext{
8 See the contribution of B. Marcos (arXiv:0805.1500) for a fuller account.

9 Indeed when such tests are performed by studying numerically the $\mathrm{N}$ dependence of results [see e.g. Diemand et al. (2004)], the temporal length of the simulation is actually changed in a manner fixed by a criterion built into the standard numerical package now used for generating initial conditions.
} 
limit. It turns out [Baertschiger et al. (2004)] that in NBS, in the phase immediately after the perturbative one described above, the evolution of the first non-linear correlations (which develop at scales well below $\ell$ ) is very well described by considering only two body interactions, and thus clearly the system at these (small) scales is not described by the Vlasov limit. In his contribution B. Marcos briefly outlines some work in progress, aimed at addressing the quantification of discreteness effects at much later times: the idea is to bound below discreteness effects in a given simulation by looking at the dispersion in measured quantities (e.g. the PS) for discretisations at fixed $N$ which differ only in the initial particle distribution used to represent uniformity. From the study of the evolution of such dispersion we should be able to get insight into how discreteness effects become more or less important as strong non-linearity sets in.

\section{Conclusions}

The question of the relation between the dynamics of a finite number of self-gravitating particles and the Vlasov limit of the same system is an open problem, which is of both theoretical and practical importance in cosmology. While we have managed to essentially solve this problem in a specific regime — early times/low amplitude fluctuations it remains a great challenge to find useful approaches to the more general problem. We have briefly described some possible such approaches and some results, which open avenues for further investigation. It would certainly be interesting to see if analagous problems encountered in other contexts as described at this conference may suggest other approaches or give insight into the problem.

I thank T. Baertschiger, A. Gabrielli, B. Marcos and F. Sylos Labini for collaboration on the work reported here.

\section{References}

J. S. Bagla, and T. Padmanabhan, Pramana 49, 161-192 (1997), astro-ph/0411730.

T. Baertschiger, M. Joyce and F. Sylos Labini, Astrophys.J. Lett. 581, L63-L66, 2002.

E. Bertschinger, Annu. Rev. Astron. Soc. 36, 599-654 (1998).

J. Binney, and S. Tremaine, Galactic Dynamics, Princeton Series in Astrophysics, Princeton, 1987.

T. Buchert and A. Dominguez, Astron.Astrophys. $438,443-460$ (2005)

A. Cooray and R. Sheth, Phys.Rept. 372, 1-129 (2002), astro-ph/0206508.

J. Diemand, B. Moore, J. Stadel, and S. Kazantzidis, Mon. Not. Roy. Astron. Soc. 348, 977 (2004), astro-ph/0304549.

A. Gabrielli, Phys. Rev. E70, 066131 (2004),

A. Gabrielli, F. Sylos Labini, M. Joyce, and L. Pietronero, Statistical Physics for Cosmic Structures, Springer-Verlag, Berlin, 2005.

M. Joyce and B. Marcos, astro-ph/0410451.

M. Joyce, B. Marcos, A. Gabrielli, T. Baertschiger, and F. Sylos Labini, Phys. Rev. Lett. 95, 011304 (2005).

B. Marcos, T. Baertschiger, M. Joyce, A. Gabrielli, and F. Sylos Labini (2006), Phys. Rev. DD73 103507 (2006). 
B. N. Miller and J.-L. Rouet, Phys. Rev. E65, 056121 (2002).

J. Peacock and S. Dodds, Mon. Not. Roy. Astron. Soc. 280, L19 (1996), astro-ph/0303031.

P. J. E. Peebles, The Large-Scale structure of the Universe, Princeton University Press, Princeton, 1980.

J.-L. Rouet, M. R. Feix and M. Navet, Vistas in Astronomy, 33, 357-370.

W. Saslaw, The Distribution of the Galaxies, Cambridge University Press, Cambridge, 2000.

R. Splinter, A. Melott, S. Shandarin and Y. Suto Astrophys.J. 497, 38-61 (1998)

H. Spohn, Large Scale Dynamics of Interacting Particle, Springer Verlag (Berlin), 1991.

G. Hinshaw et al.(the WMAP collaboration), astro-ph/0603451 